\title{
Lower and upper bounds of life expectancies and total expected longevity of a developing country
}

\author{
Ahbab Mohammad Fazle Rabbi
}

Lecturer (Statistics), Department of Applied Science, Bangladesh University of Textiles, Dhaka, Bangladesh.

\begin{abstract}
Regardless of being most used demographic indicator of longevity, life expectancy has few limitations too; and it arise mainly due to irregular mortality patterns in particular age group. This paper aims to analyze the trend of remaining life expectancies in different age groups and examines the highest observed life expectancies along with lower and upper bounds of life expectancies for Bangladesh; a country where early aged mortality is present remarkably. To estimate the lower and upper bounds of life expectancies in particular age; corresponding survival fractions have been taken into account, which gave more flexible concepts about longevity measures. Obtained results indicate decline in the gap between early aged life expectancies for both sexes in Bangladesh; which suggests that the full population, rather than just the more selected survivors, may anticipate a longer life that was previously only the domain of those who survived past the early years.
\end{abstract}

Keywords: Total Expected Longevity, Life Expectancies, Survival Function, Developing country, Bangladesh.

\section{Introduction}

In the past century, the rise of life expectancy due to mortality declines at almost all ages is one of the most remarkable achievements in the world. From a theoretical point of view, rising life expectancy means improved survival. Life table is a nice mathematical tool for Demographers to describe age-specific mortality, survival rates of a population. A central function of life tables is life expectancy by age, denoted by $e(x)$; is defined as "the average number of additional years that a survivor to age $\mathrm{x}$ will live beyond that age". ${ }^{1}$ The first of these averages, denoted as $e(0)$, is known as life expectancy at birth, a summary indicator of population health and longevity. ${ }^{1-3}$

Despite of being most used demographic indicator of longevity, life expectancy has few limitations too; and it arise mainly due to irregular mortality patterns in particular age groups. ${ }^{4}$ For instance, presence of high infant mortality creates lower life expectancy at birth than the later ages, which diminishes the unique appliance of life expectancy at birth as average longevity of a cohort. ${ }^{4,5}$ These sorts of dissimilarities have been observed for most of the under developed and developing countries; industrialized countries also faced this circumstances before a certain era. ${ }^{6}$ Similar irregularities may be seen in elder age group too, which make difficult for the demographers to comment on the longevity of a particular population. ${ }^{5,7}$ Unusual fluctuation on premature mortality rates effect the corresponding life expectancies; thus, later and ongoing gains in life expectancy are the result of mortality declines at older ages. ${ }^{5,8,9}$

Previous studies analyzed longevity in industrialized countries from selecting the highest value among all countries with available data for each year and find that, since 1840 , this record life expectancy has increased at a

\section{Practice Points}

- Presence of early aged mortality effect life expectancies; for which life expectancy at birth is not the appropriate indicator of longevity for developing countries.

- Both life expectancies at birth and highest recorded life expectancies increase significantly at Matlab HDSS.

- Variation in Demographic and epidemiologic transitions generate upper and lower bounds of life expectancies at each age/age group of life table.

- Divergence exists in life expectancies at birth and highest observed life expectancies for both sexes in Matlab HDSS.

- Analysis of maximum remaining life expectancies suggests imminent longevity in Matlab HDSS.

constant rate. ${ }^{10}$ Another study demonstrated that a straight line provided the best fit for the time trend of $e$ (0) in western industrialized countries during the second half of the $20^{\text {th }}$ century. ${ }^{11}$ These linear increase on record life expectancies was later attributed to comprises of multiple segments that correspond to distinct health transition phases. ${ }^{12}$ While the timing, pace, and trajectories of demographic and epidemiologic transitions around the world vary widely, these findings thus clearly indicate the presence of upper and lower bounds of life expectancies at each age/age group of life table. ${ }^{12-14}$

Correspondence: Ahbab Mohammad Fazle Rabbi, Lecturer (Statistics), Department of Applied Science, Bangladesh University of Textiles, Dhaka, Bangladesh. E-mail: amfrabbi@hotmail.com. 
Besides regression analysis, another common approach to compute divergence of life expectancies is to obtain interval estimation of survival function and life expectancies at particular age $x .{ }^{15}$ Chebychev's Inequality to compute interval estimates of additional years of life and analysis of measures of dispersion were suggested also to observe the divergence of life expectancies on particular age/age group. ${ }^{16,17}$ Mortality projection models also show the corresponding interval estimates for life expectancies, but like previous methods these models are also not free from effect of early aged mortality. $^{18}$

Later another method is suggested by Cohen; which does not require any regression. ${ }^{19}$ This method requires only the observed life expectancies with corresponding values of survival function at each age $x$. Simple inequalities are used to define the upper and lower bounds of life expectancies and these inequalities give bounds on $e(0)$ even without detailed knowledge of the course of mortality prior to age $x .{ }^{19}$ Application of Cohen's method on life tables of United States revealed that, the lower bound approximates $e(0)$ more closely at younger ages (up to 40 years), while the upper bound approximates $e(0)$ more closely at older ages for United States. ${ }^{19}$

Again, to overcome the constraints of life expectancies at birth as a key indicator of population longevity many modifications are suggested in previous researches. It is pointed out that, since life expectancy at birth is heavily dependent on events in early life, the time series of $e(0)$ alone was not well suited for studying the length of life in aging populations and did not distinguish between potentially important stages of mortality change. ${ }^{4}$ Maximum remaining life expectancy is a conditional measure of central tendency that reflects the effect of premature deaths while also providing a better estimate of the survivors' expected longevity than $e(0) .^{5,6,20-24}$ Maximum remaining life expectancies, record-highest remaining life expectancies along with life expectancy at birth were observed and comparisons were made across countries.; which revealed that, like other measures of longevity, the maximum remaining life expectancy and record remaining life expectancy also show upward trend. ${ }^{6}$ Nonetheless, the pace of change in any measure of longevity over time is dependent on the specific ages at which mortality improvements occur. ${ }^{25}$

However, all of these studies analyzed the longevity for developed and industrial countries only; less concern is given for developing countries where early aged mortality is present in a remarkable proportion of total mortality. Few studies focused on impact of early aged mortality on life expectancy at birth, but less concern is given on estimating effect of irregular mortality pattern over the period life table. ${ }^{26}$ Still researches needed to analyze life expectancies at older ages with analysis of trend and pattern of longevity for developing countries.

This paper aims to analyze the trend of remaining life expectancies in different age groups and examines the highest observed life expectancies along with lower and upper bounds of life expectancies for Bangladesh; a country where early aged mortality is present remarkably. To estimate the lower and upper bounds of life expectancies in particular age; corresponding survival fractions have been taken into account, which gave more flexible concepts about longevity measures. Considering these additional measures in conjunction with other measures of record longevity will allow Demographers to gain further insight into the progress of mortality reductions at specific ages over time. ${ }^{6,21}$

\section{Materials and methods}

Bangladesh, a developing country of South Asia, has not started complete vital registration yet. The vital registration and maternal and child health data gathered from Matlab, Bangladesh is utilized for current study. ${ }^{27}$ Matlab HDSS is recognized worldwide as one of the long-term demographic surveillance sites for a developing country. Since 1966, the Health and Demographic Surveillance System (HDSS) has maintained the registration of births, deaths, and migrations, in addition to carrying out periodical censuses in Matlab. Bangladesh became independent in 1971, so the life tables of 1975 to 2011 are considered for current study. The illustration is done all over the paper considering life tables separately for male and female. It should be noted that, these life tables are period life tables, cohort life tables may produce different results than this. Statistical package $R$ (version 3.0.1) and IBM SPSS Statistics (version 20) is used for statistical analysis.

Usual life tables' terminologies are carried out through this study. General mathematical relationships of abridged life tables are as follow:

Number of survivors in a particular age $x$ is $l(x)$ and number of deaths in a particular age interval $x+n$ is, $d$ $(x, n)=l(x)-l(x, n)$. At age 0 , the value of $l(x)$ is known as radix and it is considered as 1.0 in standard.

Probability of surviving in particular age $x$ to $x+n$ is, $p$ $(x, n)$ and death is $q(x, n)$, where $p(x, n)=1-q(x, n)$ and $q$ $(x, n)=d(x, n) / l(x)$. Thus, in a time interval $x+n, l(x, n)=$ $l(x) p(x, n)$ and $d(x, n)=l(x) \cdot q(x, n)$

Number of person-year lived by the cohort is, $L(x, n)=\int_{0}^{n} l(x, t) d t$

which is equivalent to

$$
L(x, n)=\frac{n l(x)+l(x, n)}{2} ;(x \geq 2)
$$

For $x<2 ; L(0)=0.201 l(0)+0.8 l(1)$ and $L(1)=0.410 l$ (1) $+0.590 l(2)$

Central death rate is defined as $m(x, n)=d(x, n) / L(x, n)$. Also,

$$
m(x)=\frac{2 q(x)}{2-q(x)}
$$


Number of person-year lived by the life table population is

$$
T(x)=\int_{0}^{\infty} L(x, t) d t
$$

which is equivalent to,

$$
T(x)=\sum_{t=0}^{\infty} L(x, t)
$$

Expectancy of life at age $x$ is, $e(x)=T(x) / l(x)$

\section{Lower and upper bounds of life expectancies}

To estimate the lower and upper bounds of life expectancies in a particular age $w$, the formal relationship of survival fraction and life expectancies derived by Cohen ${ }^{19}$ is used in this study. According to Cohen, life expectancy at birth, $e(0)$, satisfies the following inequalities for each age $x$.

$$
[x+e(x)] l(x) \leq e(0) \leq x+e(x) l(x)
$$

And for any two ages $0 \leq w \leq x \leq T$,

$$
[x-w+e(x)] \frac{l(x)}{l(w)} \leq e(w) \leq x-w+\left[e(x) \frac{l(x)}{l(w)}\right]
$$

The benefit of using these simple lower and upper bound is, these inequalities make it possible to estimate bounds for $e(0)$ without detailed knowledge of the course of mortality prior to age $x$, provided that $l(x)$ can be estimated. ${ }^{19}$ it should be noted that, Keyfitz's ${ }^{28}$ standard formula for life expectancy may be used to estimate life expectancies at any age $w$, where,

$$
e(w)=\frac{1}{l(w)} \int_{a=w}^{a=T} l(a) d a
$$

To fulfill the assumption of (2) and (3), $l(x)$ is estimated from age $x$ of life table using regression analysis. For current study, it appears from the scattered plot of the number of persons surviving at an exact age $x$ for both sexes of Matlab HDSS-2011 by age groups that $l(x)$ may be distributed by polynomial model for different ages rather than a linear model. Hence $\mathrm{n}^{\text {th }}$ degree polynomial is fitted to the values of $l(x)$ instead of linear regression model. The structure of the model is,

$$
y=a_{0}+\sum_{i=1}^{n} a_{i} \cdot x^{i}+u
$$

Where, $x$ is the exact age, $y$ is the number of survivors at age $x, a_{i}$ is the coefficients of the model (for $i=1,2,3 ; a_{0}$ is the constant term of the fitted model) and $u$ is random error. Cross validity prediction power $(C V P P) ; \rho^{2} \mathrm{CV}$; is applied here to test the stability of the fitted model. ${ }^{29}$ Symbolically,

$$
\rho_{C V}^{2}=1-\frac{(n-1)(n-2)(n+1)}{n(n-k-1)(n-k-2)}\left(1-R^{2}\right)
$$

Where ' $n$ ' is the number of observations, ' $k$ ' is the number of predictors in the model, ' $R$ ' is the correlation between observed and predicted values of the dependent variable. The shrinkage of the model is the absolute difference of $C V P P$ and $R^{2}$. Stability of $R^{2}$ of the model is defined as difference between 1 and shrinkage. ${ }^{29}$

Total expected longevity \& record high life expectancies The sum of the remaining life expectancy at age $x$ and the age $x$ at which it occurs is the total number of years that survivors to age $x$ are expected to live is referred to the total expected longevity. ${ }^{6}$ Total expected longevity for a person who attained age $x$ is denoted by $e^{T}(x)$, which is equivalent to,

$$
e^{T}(x)=x+e(x)
$$

To see the trend of life expectancy over a time series, highest observed life expectancy ${ }^{6}$ during time period $t$ is denoted by $e_{m}(t)$. Symbolically,

$$
e_{m}(t)=\max _{x}[e(x, t)]
$$

\section{Results}

The highest observed life expectancies and life expectancies at birth during 1975 to 2011 are presented below for male and female population of Matlab HDSS (Figure 1).

Presence of high infant mortality causes the lower life expectancies at birth than the later age in Bangladesh, that's why highest life expectancies may be observed after 2, 3 or 4 years of birth. Recent trend suggest very close gap between life expectancies at birth and age 1 but it will continue till $e(1)$ become equal to the inverse of Infant mortality rate. ${ }^{26}$ This trend also diminishes the unique feature of life expectancies at birth as average longevity for Bangladesh. The difference between $e(0)$ and $e(1)$ declined with decrease in infant mortality. Still the difference between $e(0)$ and $e(1)$ is over 1 , which should be zero to achieve highest life expectancy at birth instead of other ages. ${ }^{26}$

To observe the lower and upper bounds of life expectancies for Bangladesh, only data of Matlab HDSS-2011 is used. To estimate $l(x)$, polynomial model is fitted for $l$ $(x)$ considering $l(x)$ as function of age $x$. For both sexes, third degree polynomial fit the data well. The fitted models are given below respectively for male and female,

$$
\begin{aligned}
& l(x)=0.983393-0.005023 x+0.000229 x^{2}-3.289 \times 10^{-6} x^{3}(\mathrm{i}) \\
& l(x)=1.000579-0.009932 x+0.00041 x^{2}-4.635 \times 10^{-6} x^{3}(\mathrm{ii})
\end{aligned}
$$

The models are verified using Cross validity prediction power $(C V P P) ; \rho^{2} \mathrm{CV}$ is applied here to test the stability of the fitted model. The summaries of model fittings are showed in Table 1.

Both of the fitted models are stable more than $99 \%$. From t-statistics, it is found that all the parameters of the model are also highly significant. In both models, the stability of $R^{2}$ is more than $99 \%$. CVPP indicates $99 \%$ and $98 \%$ of variance are explained respectively for male and female. Estimated lower and upper bounds for 

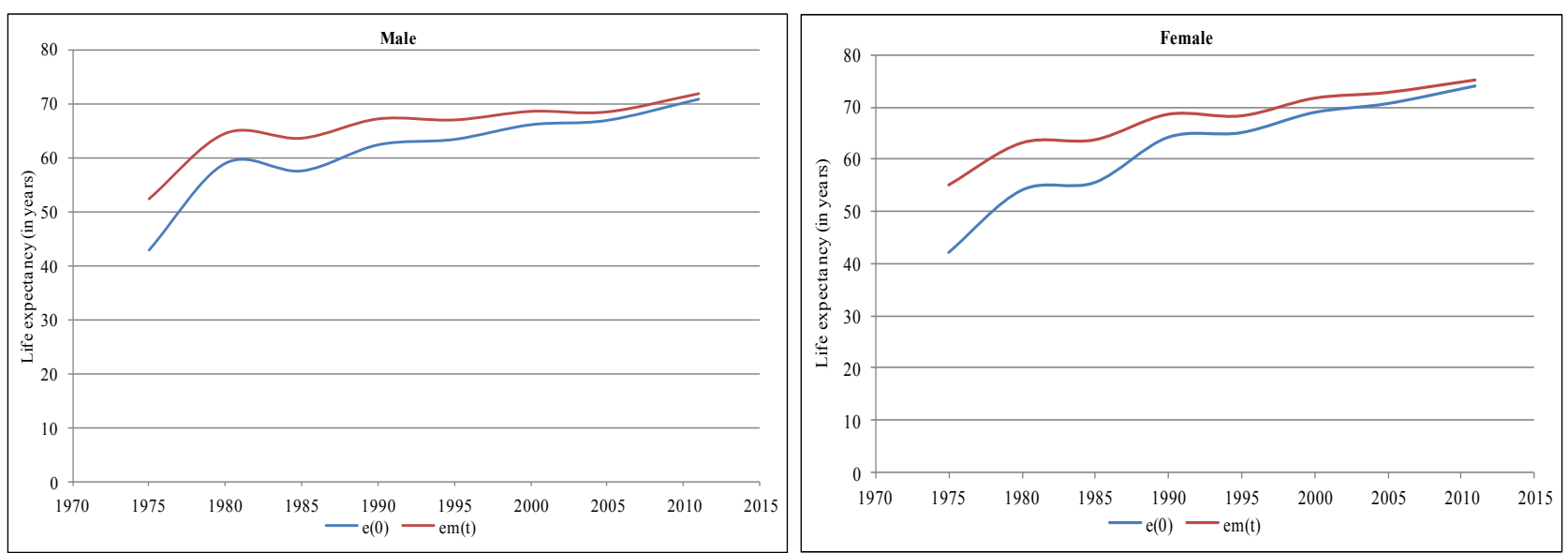

Fig 1: Trend of Life expectancies at birth and highest observed life expectancies for both sexes (Matlab HDSS, 19752011)

life expectancies for both sexes of Matlab HDSS-2011 are graphically presented in Figure 2.

For both sexes, the differences between lower and upper bounds of life expectancies are close to zero in the earlier ages. The gap between lower and upper bounds have rapid and sharp expanse from/after the age group 45 for male and for female later than 60 years. The green line represents the life expectancy at birth for both sexes. For the presence of infant mortality, the lower bounds may be found almost overlapping the lines of life expectancy at birth. Thus, both lower bounds and upper bounds approximates to $e(0)$ at younger ages more closely for both sexes, but tends to spread in the elder ages. These results demonstrate the unstable mortality pattern in later ages; similar conclusion may be drawn also from values of $l(x)$. In the following section, while analyzing the trend of total expected longevity this thing may be more clarified.

The total expected longevity, remaining life expectancies and life expectancies at birth for Matlab HDSS2011 are presented in Figure 3. Compare to Figure 1, remaining life expectancy at age one was only higher than at birth, and life expectancy at age one became the

Table 1: Summary of Model Fittings

\begin{tabular}{|c|c|c|c|c|c|c|c|c|c|c|}
\hline Model & $\mathbf{n}$ & $\mathbf{k}$ & $\mathbf{R}^{\mathbf{2}}$ & $\mathbf{F}$-statistic & $\mathbf{p}$-value & $\mathbf{\rho}_{\mathbf{C V}}$ & Shrinkage & Parameter & t-statistic & $\mathbf{p}$-value \\
\hline & & & & & & & & $\mathrm{a}_{0}$ & 170.997 & 0.0000 \\
\hline & & & & & & & & $\mathrm{a}_{1}$ & -6.351 & 0.0000 \\
\hline & & & & & & & & $\mathrm{a}_{2}$ & 9.580 & 0.0000 \\
\hline (i) & 22 & 3 & 0.998 & 2453.245 & 0.0000 & 0.996 & 0.001 & $\mathrm{a}_{3}$ & -17.200 & 0.0000 \\
\hline & & & & & & & & $\mathrm{a}_{0}$ & 78.084 & 0.0000 \\
\hline & & & & & & & & $\mathrm{a}_{1}$ & -5.636 & 0.0000 \\
\hline & & & & & & & & $\mathrm{a}_{2}$ & 7.691 & 0.0000 \\
\hline (ii) & 22 & 3 & 0.984 & 658.03 & 0.0000 & 0.982 & 0.003 & $\mathrm{a}_{3}$ & -10.886 & 0.0000 \\
\hline
\end{tabular}
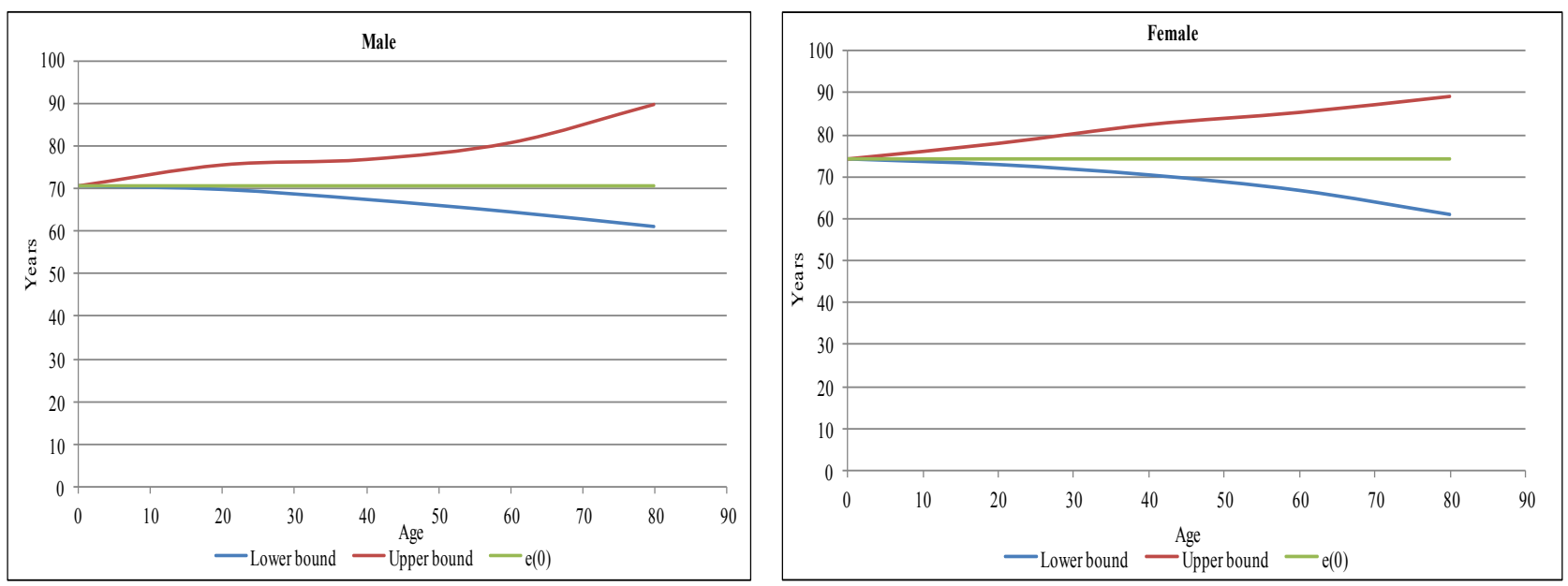

Fig 2: Lower and upper bounds for life expectancies for both sexes (Matlab HDSS-2011) 
maximum value of the remaining life expectancy in 2011. The pattern of total expected longevity at 2011 for females and males separately is quite similar, with the remaining life expectancy trajectory for males becoming monotonic slightly earlier and the trajectory for females following course a few years later. The $e^{T}(x)$ trajectory rises steeply in older ages, suggesting that survivors are no longer limited by the constraints of the mean life duration.

\section{Discussion}

The pattern over time for females and males separately is quite similar for $e(0)$ and highest observed life expectancy trajectory at Matlab HDSS. However, upward shift in record life expectancies for females becoming monotonic slightly later and the trajectory for males following course a few years earlier. Presence of early aged mortality, specially, high infant mortality causes the lower life expectancies at birth than the later age in Bangladesh. ${ }^{26}$ At 1975 , the highest life expectancies were observed at age 4 for male and 5 for female; 52.31 years and 55.07 years were the highest observed life expectancies of male and female respectively. In 1975, crossover of life expectancy at birth and other ages occurred after age 15 for male and 20 for female. ${ }^{26}$

Divergence is observed in life expectancies for both sexes in Matlab HDSS-2011 as a consequence of early aged mortality. The differences between lower and upper bounds of life expectancies are close to zero in the earlier ages. The gap between lower and upper bounds have rapid and sharp expanse from/after the age group 45 for male and for female later than 60 years; i.e., divergence occurring to female life expectancies in later age compare to male. Irregular trend in survival fraction for age groups is the reason of such pattern of upper bounds in case of male population of Matlab HDSS2011; which also affect life expectancies at higher age groups. This is a common scenario in developing/ industrial countries since decades; almost all aging population faces this scenario at phase three of demographic transition. ${ }^{14,15}$ This obtained results also indicate about epidemiological and demographic transition on
Matlab HDSS since decades.

The pattern of maximum remaining life expectancies for both sexes is quite similar; rapid upward shift is observed for both sexes, though the monotonic increase is sharper in case of male population. As illustrated in Figure 1, the $e^{T}(x)$ trajectory rises steeply in older ages, suggesting that survivors are no longer limited by the constraints of the mean life duration. This rising pattern is consistent to the next age groups, though the slope of the late-life surge diminishes somewhat as the overall average rises over time.

The reasons for the increase of total expected longevity with age is explained considering two sides. ${ }^{20}$ First, by definition remaining life expectancy is positive at all ages, as long as there are survivors alive. As a result, the longevity of a group of survivors must be higher than their age. Second, selection may be operating in the population, with persons surviving to a given age being healthier than those who died previously. The more selected survivors could thus anticipate longer lives, which may be observed also in Matlab. Nevertheless, all three indicators of current study suggest forthcoming longevity at Matlab HDSS.

Presence of early aged mortality decline the potential to analyze more insight if aging in Matlab, however, another research proved that once the effect of infant mortality is removed by conditioning on survival until age 10 , the resulting measure of variance (the standard deviation in ages at death over age 10) provides more insight into the dynamics of aging populations than the unconditional measure. ${ }^{17}$ This could be a solution for shortcoming of present researches.

\section{Conclusion}

The mortality transition reflects an epidemiological transition in morbidity patterns and causes of death for a population. Decline of the gap between early aged life expectancies for Bangladesh suggests that the full population - rather than just the more selected survivors may anticipate a longer life that was previously only the
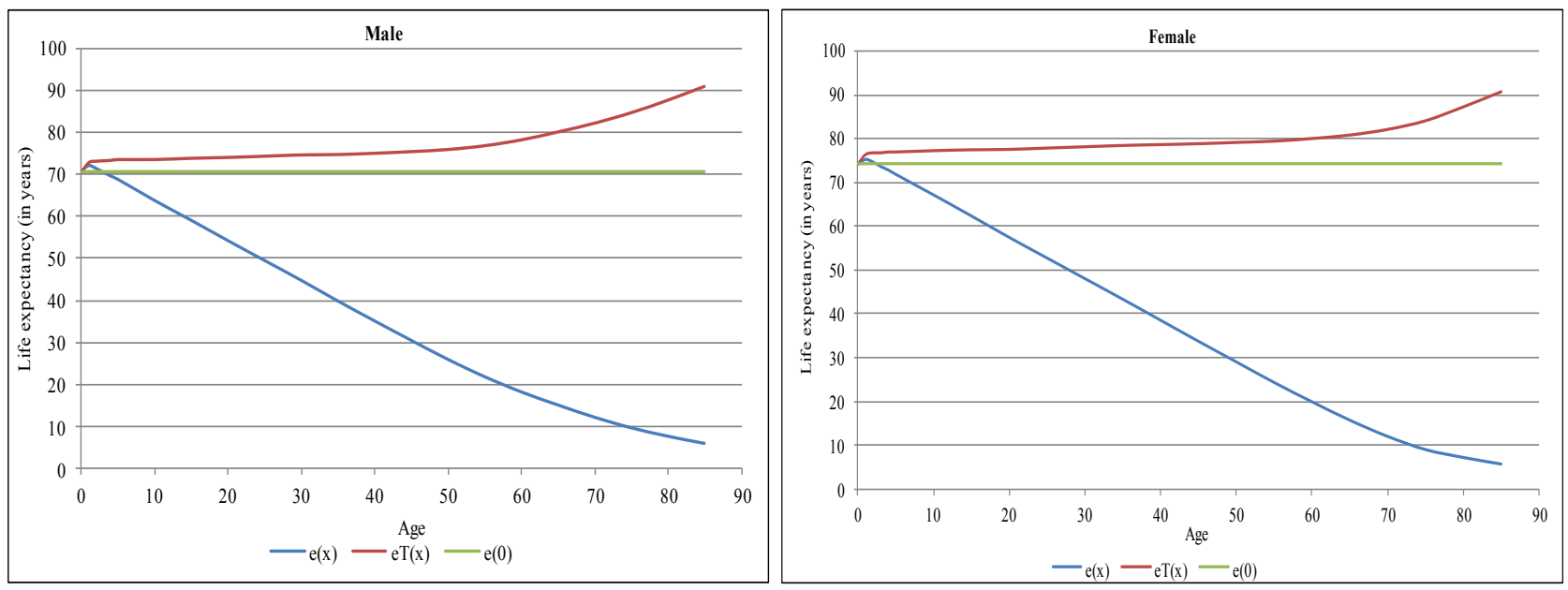

Fig 3: Total expected longevity for both sexes (Matlab HDSS-2011) 
domain of those who survived past the early years. Investigating maximum life expectancy at birth or other ages and its associated record measures will enable the policy makers to understand improvements in survival not only as markers of change over time, but also, importantly, as functions of age. By combining information about mortality reductions and the ages at which they occur, results of current study indicate a link between demographic and epidemiologic transitions and highlight the chronological connection between childhood and adult influences on the trajectory of rising life expectancy.

From the findings of current study, further research on healthy life expectancies and projection of life expectancies in later age will provide more insight of aging to the policy makers. Also, introducing improvised public health policies for aged population will be more helpful in future.

\section{References}

1. Preston SH, Heuveline P, Guillot M. Demography: Measuring and Modeling Population Processes. Malden, MA: Blackwell Publishers, 2001.

2. Dublin LI. The possibility of extending human life. Metron 1923; 3(2):175-97.

3. Dublin LI, Lotka JA. The history of longevity in the United States. Hum Bio 1934; 6(1):43-86.

4. Kannisto V. Mode and dispersion of the length of life. Popul Engl Sel 2001; 13(1):159-71.

5. Vaupel JW. How change in age-specific mortality affects life expectancy. Popul Stud 1986; 40 (1):147-57.

6. Romo CV, Engelman M. Maximum life expectancies: Revisiting the best practice trends. Genus 2009; LXV(1):59-79.

7. Romo CV, Becker S. The crossover between life expectancies at birth and at age one: The imbalance in the life table. Demo Res 2011; 24:113-44.

8. Horiuchi S. Assessing the effects of mortality reduction on population ageing. Popul Bull UN 1991; 31(2):38-51.

9. Wilmoth JR, Robine JM. The world trend in maximum life span. Popul Dev Rev 2003; 29 (Suppl): 239-257.

10. Oeppen J, Vaupell JW. Broken limits to life expectancy. Science 2002; 296:1029-31.

11. White KM. Longevity advances in high-income countries, 1955-96. Popul Dev Rev 2002; 28 (1):59-76.

12. Vallin J, Meslé F. The segmented trend line of highest life expectancies. Popul Dev Rev 2009; 35(1):159-87.

13. Riley JC. The timing and pace of health transition around the world. Popul Dev Rev 2005; 31(4):741-64.

14. Cheung S, Robine J, Tu E, Caselli G. Three dimensions of the survival curve: Horizontalization, verticalization, and longevity extension. Demography 2005; 42(2):243-58.

15. James C, Ciecka P. Life expectancy and the properties of survival data. Litig Econ (Digest) Rev 1996; 1(2):19-33.

16. DeGroot M. Probability and Statistics, 2nd ed., Reading, Massachusetts: Addison Wesley, 1989.

17. Edwards RD, Tuljapurkar S. Inequality in life spans and a new perspective on mortality convergence across industrialized countries. Popul Dev Rev 2005; 31(4):645-74.

18. Shang HL, Booth H, Hyndman R. Point and interval forecasts of mortality rates and life expectancy: A comparison of ten principal component methods. Demo Res 2011; 25:173-214.

19. Cohen JE. Life expectancy: Lower and upper bounds from surviving fractions and remaining life expectany. Demo Res 2011; 24:251-6.

20. Mirowsky J. Subjective life expectancy in the US: Correspondence to actuarial estimates by age, sex and race. Soc Sci Med 1999; 49:967-79.

21. Nusselder WJ, Mackenbach JP. Lack of improvement of life expectancy at advanced ages in The Netherlands. Int $J$ Epidemiol 2000; 29:140-8.

22. Wilmoth JR, Lundstrom H. Extreme longevity in five countries. Eur J Popul 1996;12:63-93.

23. Wilmoth JR, Horiuchi S. Rectangularization revisited: Variability of age at death within human populations. Demography 1999; 36:475-95.

24. Robine JM. Redefining the stages of the epidemiological transition by a study of the dispersion of life spans: the case of France. Popul Engl Sel 2001; 13(1):173-94.

25. Wilmoth JR. (2000), Demography of longevity: Past, present and future trends. Exp Gerontol 2000; 35(9-10):1111-29.

26. Fazle Rabbi AM. Imbalance in Life Table: Effect of Infant Mortality on Lower Life Expectancy at Birth. J Sci Res 2013; 5(3):479-88.

27. ICDDR,B. Health and Demographic Surveillance System-Matlab, v. 45. Registration of health and demographic events 2011. Scientific Report No. 121. Dhaka: ICDDR,B, 2012.

28. Keyfitz N. Applied Mathematical Demography. 1st ed. New York: John Wiley, 1977.

29. Stevens J. Applied Multivariate Statistics for the Social Sciences, Third Edition, New Jersey: Lawrence Erlbaum Associates, Inc,1996. 